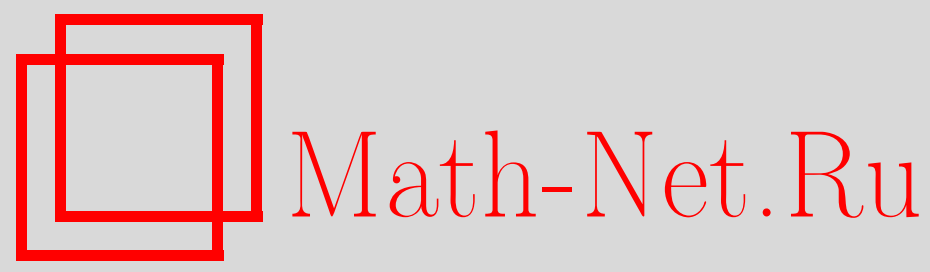

Н. М. Тимофеев, М. Б. Хрипунова, О функции концентрации для аддитивных функций со специальным весом, $M a$ тем. заметки, 2004, том 76, выпуск 2, 265-285

DOI: https://doi.org/10.4213/mzm105

Использование Общероссийского математического портала Math-Net.Ru подразумевает, что вы прочитали и согласны с пользовательским соглашением http://www.mathnet.ru/rus/agreement

Параметры загрузки:

IP : 52.23 .180 .231

26 апреля 2023 г., $16: 24: 42$

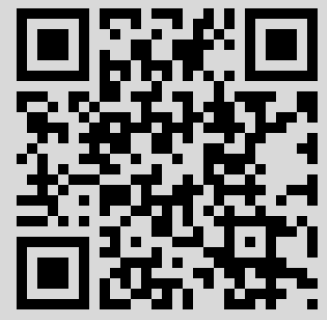




\section{О ФУНКЦИИ КОНЦЕНТРАЦИИ ДЛЯ АДДИТИВНЫХ ФУНКЦИЙ СО СПЕЦИАЛЬНЫМ ВЕСОМ}

\section{Н.М. Тимофеев, М.Б. Хрипунова}

Пусть $g(n)$ - аддитивная функция принимающая вещественные значения,

$$
W(N)=4+\min _{\lambda}\left(\lambda^{2}+\sum_{p<N} \frac{1}{p} \min \left(1,(g(p)-\lambda \log p)^{2}\right)\right), \quad E(N)=4+\sum_{\substack{p<N, g(p) \neq 0}} \frac{1}{p}
$$

В работе доказано существование постоянных $C_{1}, C_{2}$ такие, что справедливы неравенства

$$
\begin{aligned}
& \sup _{a}\left|\left\{n, m, k: m, k \in \mathbb{Z}, n \in \mathbb{N}, n+m^{2}+k^{2}=N, g(n) \in[a, a+1)\right\}\right| \leqslant \frac{C_{1} N}{\sqrt{W(N)}}, \\
& \sup _{a}\left|\left\{n, m, k: m, k \in \mathbb{Z}, n \in \mathbb{N}, n+m^{2}+k^{2}=N, g(n)=a\right\}\right| \leqslant \frac{C_{2} N}{\sqrt{E(N)}} .
\end{aligned}
$$

Доказанные оценки точны по порядку.

Библиография: 10 названий.

1. Введение и формулировка результатов. Пусть $g(n)$ - аддитивная функция, $\tau(n)$ - число делителей $n$ и $r(n)$ - число представлений $n$ в виде суммы квадратов двух целых чисел. В предыдущей работе, используя результаты статьи Ружи [1], идеи и методы работ Эллиота [2] и Тимофеева [3], была доказана следующая

Теорема [4, теорема 1]. Существует абсолютная постоянная $C$ такая, что для любой аддитивной функиии $g(n)$ справедливо неравенство

$$
\sup _{a} \sum_{\substack{n<N, g(n) \in[a ; a+1)}} \tau(N-n) \leqslant \frac{C N \log (2 N)}{\sqrt{W(N)}}
$$

$2 \partial e$

$$
W(N)=4+\min _{\lambda}\left(\lambda^{2}+\sum_{p<N} \frac{1}{p} \min \left(1,(g(p)-\lambda \log p)^{2}\right)\right) .
$$

Работа выполнена при финансовой поддержке Российского фонда фундаментальных исследований, грант № 02-01-00368. 
В теореме 1 [4] доказано, что

$$
\sup _{a}|\{n, m, k: n, m, k \in \mathbb{N}, N=n+m k, g(n) \in[a ; a+1)\}| \leqslant C \frac{N \log (2 N)}{\sqrt{W(N)}} .
$$

Из этой теоремы легко следует (см. следствие 1 [4])

$$
\sup _{a}|\{n, m, k: n, m, k \in \mathbb{N}, N=n+m k, g(n)=a\}| \leqslant C \frac{N \log (2 N)}{\sqrt{E(N)}},
$$

где

$$
E(N)=\sum_{\substack{p<N, g(p) \neq 0}} \frac{1}{p}+4
$$

Целью настоящей работы является доказательство следующего результата.

Теорема 1. Существует абсолютная постоянная $C$ такая, что для любой аддитивной функиии $g(n)$ справедливо неравенство

$$
\sup _{a} \sum_{\substack{n<N, g(n) \in[a ; a+1)}} r(N-n) \leqslant C \frac{N}{\sqrt{W(N)}} .
$$

В теореме 1 оценено число решений следующей бинарной задачи

$$
N=n+m^{2}+k^{2}, \quad \text { где } g(n) \in[a ; a+1), \quad m, k \in \mathbb{Z},
$$

причем полученная оценка равномерна по $a$ и $g$.

Из теоремы 1 следует (см. [4, доказательство следствия 1])

СлЕДСТВИЕ 1. Существует абсолютная постоянная, с которой

$$
\sup _{a} \sum_{\substack{n<N, g(n)=a}} r(N-n) \leqslant C \frac{N}{\sqrt{E(N)}}
$$

Здесь, так же как и в работе [4], мы доказываем наиболее сложный случай. Несколько проще можно доказать следующее утверждение.

Теорема 2. Пусть $b \neq 0$ - иелое число. Существует постоянная, зависящая только от $b$ такая, что для любой аддитивной функиии $g(n)$ справедливо неравенство

$$
\sup _{a} \sum_{\substack{0<n+b \leqslant N, g(n) \in[a ; a+1)}} r(n+b) \leqslant C \frac{N}{\sqrt{W(N)}} .
$$

Из этой теоремы вытекает

СлЕДСТВИЕ 2. Существует постоянная $C$, зависящая только от b, такая, что для любой аддитивной функции $g(n)$ справедливо неравенство

$$
\sup _{a} \sum_{\substack{0<n+b \leqslant N, g(n)=a}} r(n+b) \leqslant C(b) \frac{N}{\sqrt{E(N)}} .
$$


Можно показать, что полученные результаты, вообще говоря, неулучшаемы. Действительно, теорема 1 [5] утверждает, что при $k \leqslant(2-\varepsilon) \log \log N$

$\nu(N, k):=\left|\left\{n: N=n+m^{2}+l^{2}, m, l \in \mathbb{Z}, \Omega(n)=k\right\}\right| \sim C(N, k) \frac{N}{\log N} \frac{(\log \log N)^{k-1}}{(k-1) !}$,

где $\Omega(n)$ - число простых делителей $n$ с учетом их кратности. Если $k=[\log \log N]$, то $C(N, k) \geqslant c_{1}>0$ и из формулы Стирлинга следует, что

$$
\frac{N}{\log N} \frac{(\log \log N)^{k-1}}{(k-1) !} \geqslant c_{2} \frac{N}{\sqrt{\log \log n}}, \quad c_{2}>0
$$

откуда

$$
\nu(N, k) \geqslant c_{1} c_{2} \frac{N}{\sqrt{\log \log N}} .
$$

В случае $g(n)=\Omega(n)$ мы имеем $W(N)=E(N)=\log \log N$, т.е. оценки теоремы 1 и следствия 1 точны по порядку.

2. Вспомогательные результаты. Обозначим через $p(n), q(n)$ наибольший и наименьший простые делители $n$ соответственно. Положим $p(1)=q(1)=1$.

Лемма 1. Пусть $2 \leqslant r \leqslant x$. Тогда

$$
|\{n: n \leqslant x, p(n) \leqslant r\}| \ll x \exp \left(-\frac{\log x}{2 \log r}\right) .
$$

Доказательство этой леммы приведено, например, в [6] (см. главу III.5, теорему 1).

Лемма 2. Пусть $r \geqslant 2, z \geqslant 2$. Тогда

$$
\sum_{\substack{p(n) \leqslant r, n>z}} \frac{1}{n} \ll \exp \left(-\frac{1}{2} \frac{\log z}{\log r}\right) \log r, \quad \sum_{\substack{p(n) \leqslant r, n>z}} \frac{1}{\varphi(n)} \ll \exp \left(-\frac{1}{4} \frac{\log z}{\log r}\right) \log ^{2} r .
$$

ДоКАЗАТЕЛЬСТВо. Используя лемму 1 , находим

$$
\begin{aligned}
\sum_{\substack{p(n) \leqslant r, n>z}} \frac{1}{n} & \leqslant \sum_{k \geqslant 0} \frac{1}{2^{k} z}\left|\left\{n: n \leqslant 2^{k+1} z, p(n) \leqslant r\right\}\right| \\
& \ll \sum_{k \geqslant 0} \exp \left(-\frac{\log 2}{2} \frac{k+1}{\log r}\right) \exp \left(-\frac{1}{2} \frac{\log z}{\log r}\right) \ll \exp \left(-\frac{1}{2} \frac{\log z}{\log r}\right) \log r .
\end{aligned}
$$

Докажем второе соотношение леммы 2. Пусть $y=\exp (\log z /(4 \log r))$. Имеем

$$
\begin{aligned}
\sum_{\substack{p(n) \leqslant r, n>z}} \frac{n}{\varphi(n)} \frac{1}{n} & =\sum_{\substack{p(n) \leqslant r, n>z}} \frac{1}{n} \sum_{\delta \mid n} \frac{\mu^{2}(\delta)}{\varphi(\delta)} \\
& \leqslant \sum_{\delta \leqslant y} \frac{\mu^{2}(\delta)}{\delta \varphi(\delta)} \sum_{\substack{p(n) \leqslant r, n>z / y}} \frac{1}{n}+\frac{1}{y} \sum_{p(\delta) \leqslant r} \frac{\mu^{2}(\delta)}{\varphi(\delta)} \sum_{p(n) \leqslant r} \frac{1}{n} \\
& \ll \exp \left(-\frac{1}{4} \frac{\log z}{\log r}\right) \log ^{2} r .
\end{aligned}
$$

Лемма 2 доказана. 
ЛЕмма 3. Пусть $\chi$ - неглавный характер Дирихле по модулю $4 u \log m \ll r \log r$, $r \geqslant 2$. Тогдa

$$
\sum_{\substack{n \leqslant y,(n, m)=1, q(n)>r}} \frac{\chi(n)}{n}=1+O\left(\exp \left(-\frac{\log y}{2 \log r}\right) \log r+\frac{\log m}{r}\right)
$$

ДокАЗАТЕльСтво. Обозначим $m(r, y)$ - произведение простых делителей $m$ таких, что $r<p \leqslant y$. Тогда имеем

$$
\sum:=\sum_{\substack{n \leqslant y,(n, m)=1, q(n)>r}} \frac{\chi(n)}{n}=\sum_{\substack{l \leqslant y, p(l) \leqslant r}} \frac{\chi(l) \mu(l)}{l} \sum_{\substack{\delta \leqslant y / l, \delta \mid m(r, y)}} \frac{\chi(\delta) \mu(\delta)}{\delta} \sum_{n \leqslant y /(l \delta)} \frac{\chi(n)}{n} .
$$

Учитьвая, что

$$
\sum_{n \leqslant t} \frac{\chi(n)}{n}=\prod_{p}\left(1-\frac{\chi(p)}{p}\right)^{-1}+O\left(\frac{1}{t}\right)
$$

получаем

$$
\begin{aligned}
\sum & =\sum_{\substack{l \leqslant y, p(l) \leqslant r}} \frac{\chi(l) \mu(l)}{l} \sum_{\substack{\delta \leqslant y / l, \delta \mid m(r, y)}} \frac{\chi(\delta) \mu(\delta)}{\delta} \prod_{p}\left(1-\frac{\chi(p)}{p}\right)^{-1}+O\left(\frac{1}{y} \sum_{\substack{l \leqslant y, p(l) \leqslant r}} \sum_{\substack{\delta \leqslant y / l, \delta \mid m(r, y)}} 1\right) \\
& =\prod_{p>r}\left(1-\frac{\chi(p)}{p}\right)^{-1}+O\left(\sum_{\substack{l>y, p(l) \leqslant r}} \frac{1}{l}+\sum_{\substack{l \leqslant y, p(l) \leqslant r}} \frac{1}{l}\left|\sum_{\delta \mid m(r, y)} \frac{1}{\delta}-1\right|+\frac{1}{y} \sum_{\substack{l \leqslant y, p(l) \leqslant r}} 1\right) .
\end{aligned}
$$

Произведение, стоящее в правой части последнего равенства, будет равно

$$
\exp \left(-\sum_{p>r} \log \left(1-\frac{\chi(p)}{p}\right)\right)=\exp \left(\sum_{p>r} \frac{\chi(p)}{p}\right)+O\left(\frac{1}{r}\right) .
$$

Так как (см., например, [7, следствие 1, с. 154])

$$
\sum_{p \leqslant t} \chi(p)=\sum_{\substack{p \leqslant t, p \equiv 1(\bmod 4)}} 1-\sum_{\substack{p \leqslant t, p \equiv 3(\bmod 4)}} 1=O(t \exp (-c \sqrt{\log t})),
$$

где $c>0$, то после суммирования по Абелю получим, что произведение равно

$$
1+O(\exp (-c \sqrt{\log r})), \quad c>0 .
$$

Учитьвая, что

$$
\sum_{\delta \mid m(r, y)} \frac{1}{\delta}-1 \ll \exp \left(\sum_{\substack{p \mid m \\ p>r}} \frac{1}{p}\right)-1 \ll \frac{\log m}{r \log r}
$$

применив леммы 1 и 2 , находим

$$
\sum=1+O\left(\exp \left(-\frac{1}{2} \frac{\log y}{\log r}\right) \log r+\frac{\log m}{r}\right) .
$$

Лемма 3 доказана.

Из теоремы 3.4 [8] следует 
Лемма 4. Пусть $k \leqslant x^{\alpha}, 0<\alpha<1,2 \leqslant z \log z \leqslant \sqrt{x^{1-\alpha}}$. Тогдa

$$
|\{n: n \equiv l(\bmod k), p(n) \geqslant z\}| \ll \frac{x}{\varphi(k) \log z} .
$$

Обозначим $H(x, y, z)$ - число натуральных чисел $n \leqslant x$, имеющих хотя бы один делитель $d, y<d \leqslant z$. Тогда из теоремы 21 [9] (см. также [9, неравенство 2.2, с. 28]) вытекает

ЛЕмма 5. $Е с л u \quad 3 \leqslant y \leqslant \sqrt{x}$, mo

$$
H(x, y, 2 y) \ll \frac{x}{(\log y)^{\beta} \sqrt{\log \log y}},
$$

где $\beta=1-(1+\log \log 2) / \log 2=0.08607 \ldots$

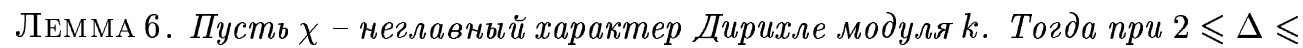
$x^{1 / 10}, y(x, m)=m \Delta / \sqrt{x}$ или $\sqrt{x} / \Delta$ имеем

$$
\left.\sum_{m \leqslant x}\right|_{y(x, m)<d \leqslant \sqrt{x} \Delta,} \chi(d) \mid \ll x \frac{(\log (\Delta \log x))^{3 / 2}}{(\log x)^{\beta / 2}(\log \log x)^{1 / 2}} .
$$

ДокАЗАТЕльство. Для доказательства мы воспользуемся методом Хооли (см. [10, глава 5]). Применив неравенство Коши получим

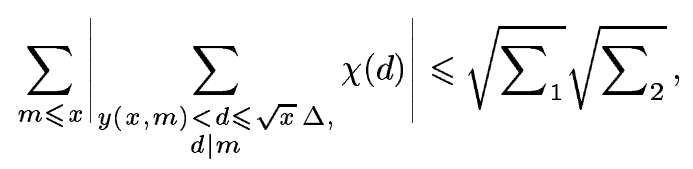

где

$$
\sum_{1}:=H\left(x, \frac{\sqrt{x}}{\Delta}, \sqrt{x} \Delta\right), \quad \sum_{2}=\sum_{m \leqslant x}\left|\sum_{\substack{m(x, m)<d \leqslant \sqrt{x} \Delta, d \mid m}} \chi(m)\right|^{2} .
$$

Для $\sum_{1}$ имеем

$$
\begin{aligned}
\sum_{1} & \leqslant H\left(x, \frac{\sqrt{x}}{\Delta}, \sqrt{x}\right)+H(x, \sqrt{x}, \sqrt{x} \Delta) \\
& \leqslant 2 H\left(x, \frac{\sqrt{x}}{\Delta^{2} \log x}, \sqrt{x}\right)+\frac{x}{\Delta \log x} \\
& \leqslant 2 \sum_{0 \leqslant j \leqslant J} H\left(x, 2^{j} \frac{\sqrt{x}}{\Delta^{2} \log x}, 2^{j+1} \frac{\sqrt{x}}{\Delta^{2} \log x}\right)+\frac{x}{\Delta \log x}
\end{aligned}
$$

где $J=O(\log (\Delta \log x))$. Применив лемму 5, получим

$$
\sum_{1} \ll \frac{x(\log (\Delta \log \log x)}{(\log x)^{\beta} \sqrt{\log \log x}} .
$$


После возведения в квадрат и перестановки порядка суммирования получим при $y(x, m)=m \Delta / \sqrt{x}$

$$
\sum_{2}=\sum_{\substack{\sqrt{x} \\ \Delta} d_{1}, d_{2} \leqslant \sqrt{x} \Delta,} \chi\left(d_{1}\right) \bar{\chi}\left(d_{2}\right) \sum_{\substack{m \leqslant z\left(x, d_{1}, d_{2}\right),\left[d_{1}, d_{2}\right] \leqslant x}} 1
$$

где

$$
z\left(x, d_{1}, d_{2}\right)=\min \left(\frac{d_{1} \sqrt{x}}{\Delta}, \frac{d_{2} \sqrt{x}}{\Delta}, x\right)=\min \left(\frac{d_{1} \sqrt{x}}{\Delta}, \frac{d_{2} \sqrt{x}}{\Delta}\right) .
$$

Следовательно, имеем

$$
\sum_{2}=\sum_{\substack{\sqrt{x} \\ \frac{\sqrt{x}}{\Delta}<d_{1}, d_{2} \leqslant \sqrt{x} \Delta,\left[d_{1}, d_{2}\right] \leqslant x}}\left(\chi\left(d_{1}\right) \bar{\chi}\left(d_{2}\right) \frac{z\left(x, d_{1}, d_{2}\right)}{\left[d_{1}, d_{2}\right]}+O(1)\right) .
$$

Пусть $\delta=\left(d_{1}, d_{2}\right)$. Тогда $d_{1}=\delta m_{1}, d_{2}=\delta m_{2},\left(m_{1}, m_{2}\right)=1,\left[d_{1}, d_{2}\right]=\delta m_{1} m_{2}$ и

$$
\sum_{2}=\sum_{\delta \leqslant \sqrt{x} \Delta} \sum_{\substack{\frac{\sqrt{x}}{\delta \Delta}<m_{1}, m_{2} \leqslant \frac{\sqrt{x} \Delta}{\delta}, \delta m_{1} m_{2} \leqslant x,\left(m_{1}, m_{2}\right)=1}}\left(\frac{\chi\left(m_{1}\right) \bar{\chi}\left(m_{2}\right)}{\delta m_{1} m_{2}} z\left(x, \delta m_{1}, \delta m_{2}\right)+O(1)\right) .
$$

Сумма, которая получается при суммировании $O(1)$, будет

$$
\ll x \sum_{\delta \leqslant \Delta^{4}} \sum_{\sqrt{x} /(\delta \Delta) \leqslant m_{1} \leqslant \sqrt{x} \Delta / \delta} \frac{1}{\delta m_{1}}+x \Delta^{2} \sum_{\delta>\Delta^{4}} \frac{1}{\delta^{2}} \ll x \log ^{2} \Delta .
$$

Суммирование по Абелю дает

$$
\sum_{u<m \leqslant v} \frac{\chi(m)}{m} \ll \frac{1}{u}
$$

Поэтому

$$
\sum_{\substack{u<m \leqslant v,(m, a)=1}} \frac{\chi(m)}{m}=\sum_{d \mid a} \frac{\chi(d) \mu(d)}{d} \sum_{u / d<m \leqslant v / d} \frac{\chi(m)}{m} \ll \frac{\tau(a)}{u} .
$$

Учитывая (2), получим, что первая сумма оценивается сверху через

$$
\begin{aligned}
2 \mid & \sum_{\delta \leqslant \sqrt{x} \Delta \sqrt{x} /(\delta \Delta)<m_{1}<m_{2} \leqslant \sqrt{x} \Delta / \delta,} \chi\left(m_{1}\right) \frac{\sqrt{x}}{\Delta} \frac{\bar{\chi}\left(m_{2}\right)}{m_{2}} \mid+\sqrt{x} \Delta \\
& \ll \frac{\sqrt{x}}{\Delta} \sum_{\delta \leqslant \sqrt{x} m_{2} \leqslant x,\left(m_{1}, m_{2}\right)=1} \sum_{m_{1} \leqslant \frac{\sqrt{x} \Delta}{\delta}} \frac{\tau\left(m_{1}\right)}{m_{1}}+\sqrt{x} \Delta \\
& \ll \frac{\sqrt{x}}{\Delta}\left(\sum_{\delta \leqslant \frac{\sqrt{x}}{\Delta \log ^{2} x}} \log ^{2} x+\sqrt{x} \Delta \log ^{2}(\Delta \log x)\right) \\
& \ll x \log ^{2}(\Delta \log x) .
\end{aligned}
$$


Таким образом,

$$
\sum_{2} \ll x \log ^{2}(\Delta \log x)
$$

и поэтому, учитьвая $(1)$, получим утверждение леммы 6 . Если $y(x, m)=\sqrt{x} / \Delta$, то $z\left(x, d_{1}, d_{2}\right)=x$, и снова применив $(2)$, получим

$$
\begin{aligned}
\sum_{2} & \ll x \sum_{\delta \leqslant \sqrt{x} \Delta} \frac{1}{\delta} \sum_{\sqrt{x} /(\delta \Delta) \leqslant m_{1} \leqslant \sqrt{x} \Delta / \delta} \frac{\tau\left(m_{1}\right)}{m_{1}^{2}}+x \log ^{2} \Delta \\
& \ll x\left(\sum_{\delta \leqslant \frac{\sqrt{x}}{\Delta^{4} \log ^{4} x}} \frac{1}{\delta} \frac{1}{\Delta^{3} \log ^{4} x} \log ^{2} x+\sum_{\frac{\sqrt{x}}{\Delta^{4} \log ^{4} x} \leqslant \delta \leqslant \sqrt{x} \Delta} \frac{1}{\delta}+\log ^{2} \Delta\right) \\
& \ll x \log ^{2}(\Delta \log x) .
\end{aligned}
$$

То есть, учитывая (1), снова получаем утверждение леммы. Лемма 6 доказана.

ЛЕмма 7. Пусть $\left\{a_{n}\right\},\left\{b_{m}\right\}$ - последовательности комплексных чисел такие, что $a_{n}=0$ при $n \leqslant z_{1}, b_{m}=0$ при $m \leqslant z_{2}, d_{1}, d_{2}$ - натуральные числа, причем $p\left(d_{1}\right) \leqslant z, q\left(d_{2}\right)>z$. Тогда

$$
\begin{aligned}
\Delta(Q):= & \sum_{d=d_{1} d_{2} \leqslant Q} \max _{(a, d)=1}\left|\sum_{\substack{n m \leqslant x, n m \equiv a(\bmod d)}} a_{n} b_{m}-\frac{1}{\varphi\left(d_{2}\right)} \sum_{\substack{n m \leqslant x,\left(n m, d_{2}\right)=1, n m \equiv a\left(\bmod d_{1}\right)}} a_{n} b_{m}\right| \\
\ll & \left(\frac{1}{\log ^{A} x}\left(\sum_{k \leqslant 2 x}\left(\sum_{n m=k}\left|a_{n} b_{m}\right|\right)^{2}\right)^{1 / 2}\right. \\
& \left.+\left(\sum_{|k-x| \leqslant x /\left(\log ^{A} x\right)}\left(\sum_{n m=k}\left|a_{n} b_{m}\right|^{2}\right)\right)^{1 / 2}\right)\left(Q+\sqrt{x} \log ^{3 / 2} x\right) \\
& +(\log \log x) \max _{y \leqslant x}\left(\frac{1}{x} \sum_{n \leqslant y}\left|a_{n}\right|^{2} \sum_{m \leqslant x / y}\left|b_{m}\right|^{2}\right)^{1 / 2} \\
& \times\left(Q \sqrt{x} \log \frac{x}{z_{1} z_{2}}+\left(\frac{x}{\sqrt{z_{2}}}+\frac{x}{\sqrt{z_{1}}}\right) \log ^{2} Q+\frac{x}{z}(\log Q) \log \frac{x}{z_{1} z_{2}}\right)
\end{aligned}
$$

где $A$ - любая положительная постоянная.

Доказательство этой леммы приведено в работе [4].

Нам потребуется теорема Халоса. Мы приведем ее в форме, доказанной в [6, глава 4, следствие 6.3].

ЛЕмма 8. Существует абсолютная постоянная с такая, что для любой мультипликативной функиии $f,|f(n)| \leqslant 1$, справедливо неравенство

$$
\left|\sum_{n \leqslant x} f(n)\right| \leqslant c x\left(\exp \left(-\frac{1}{2} \min _{|t| \leqslant T} \sum_{p \leqslant x} \frac{1-\operatorname{Re} f(p) p^{-i t}}{p}\right)+\frac{1}{\sqrt{T}}\right) .
$$

Следующие две леммы доказаны в [4, леммы 3,4$]$. 
ЛЕмма 9. Пусть $|f(p)| \leqslant 1$ для всех простых чисел р. Предположим, что найдется примитивный характер модуля $\delta, \delta \leqslant \log ^{2} N$, и вещественное число $\lambda_{0}$, $\left|\lambda_{0}\right| \leqslant \log ^{2} N$, такие, что

$$
\sum_{p \leqslant N} \frac{1}{p}\left(1-\operatorname{Re} f(p) p^{-i \lambda_{0}} \chi_{\delta}(p)\right) \leqslant \frac{1}{100} \log \log N .
$$

Тогда для всех характеров Дирихле $\chi_{d} c d \leqslant \log ^{2} N$, не порожденных $\chi_{\delta}, n p u$ $|\lambda| \leqslant \log ^{2} N$ справедливо неравенство

$$
\sum_{p \leqslant N} \frac{1}{p}\left(1-\operatorname{Re} f(p) p^{-i \lambda} \chi_{d}(p)\right) \geqslant \frac{1}{2} \log \log N+O(1) .
$$

ЛЕмма 10. Существует абсолютная постоянная $c>0$ такая, что для любой мультипликативной функиии $f,|f(n)| \leqslant 1$, выполняется неравенство

$$
\begin{aligned}
& \left|\sum_{\substack{n \leqslant x,(n, N)=1}} f(n)-\frac{\varphi(N)}{N} \sum_{n \leqslant x} f(n)\right| \\
& \quad \leqslant c x\left(\sum_{d \mid N} \frac{1+\log d}{d}\right)^{1 / 2}(\log x)^{-1 / 4} \exp \left(\frac{1}{2} \sum_{p \leqslant x} \frac{1}{p}|f(p)-1|\right) .
\end{aligned}
$$

И, наконец, нам потребуется теорема Ружи [1], аналогом которой являются результаты работ [2]-[4] и настоящей работы.

Лемма 11. Существует абсолютная постоянная такая, что для любой аддитивной функиии справедливо неравенство

$$
\frac{1}{x} \sup _{a}|\{n: n \leqslant x, a \leqslant g(n)<a+1\}| \leqslant \frac{c}{\sqrt{W(x)}},
$$

$2 \partial e$

$$
W(x)=4+\min _{\lambda}\left(\lambda^{2}+\sum_{p \leqslant x} \frac{1}{p} \min \left(1,(g(p)-\lambda \log p)^{2}\right)\right) .
$$

3. Доказательство теоремы 1. Обозначим $P_{1}$ - множество натуральных чисел, все простые делители которых сравнимы с единицей по модулю 4 , и $P_{3}$ - множество натуральных чисел, которые могут делиться на 2 и на простые сравнимые с 3 по модулю 4 . Положим далее $\mathscr{L}=\log N$. Известно, что $r(n)=4 r_{1}(n), r_{1}(n)=\sum_{d \mid n} \chi(d), \chi-$ неглавный характер Дирихле по модулю 4. Имеем

$$
S(N):=\sum_{\substack{n<N, g(n) \in[a ; a+1)}} r(N-n)=4 \sum_{\delta \in P_{3}} r_{1}(\delta) \sum_{n \in A(N, g, a, \delta)} r_{1}\left(\frac{N-n}{\delta}\right)
$$

где

$$
A(N, g, a, \delta)=\left\{n: n<N, n \equiv N(\bmod \delta), \frac{N-n}{\delta} \in P_{1}, g(n) \in[a ; a+1)\right\}
$$


Заметим, что если $m \in P_{1}$, то $r_{1}(m)=\tau(m)$ и оценим ту часть суммы $S(N)$, в которой $\delta \geqslant u=\log ^{3} \mathscr{L}$. Учитьвая, что (см., например, [9, теорема 0.1])

$$
\sum_{\substack{m \in P_{1} \\ m \leqslant x}} \tau(m) \ll \frac{x}{\log x} \prod_{p \equiv 1}\left(1+\sum_{\substack{(\bmod 4) \\ p \leqslant x}}^{\infty} \frac{\tau\left(p^{r}\right)}{p^{r}}\right) \ll x,
$$

получим, что эта часть суммы $S(N)$ будет

$$
\ll \sum_{\delta \geqslant \log ^{3} \mathscr{L}} r_{1}(\delta) \frac{N}{\delta} \ll \frac{N}{\log \mathscr{L}} \prod_{p}\left(1+\sum_{r=1}^{\infty} \frac{r_{1}\left(p^{r}\right)}{p^{2 r / 3}}\right) \ll \frac{N}{\log \mathscr{L}}
$$

Tак как $\tau(m \cdot n) \leqslant \tau(m) \cdot \tau(n)$, то

$$
\begin{aligned}
S(N) \leqslant & 4 \sum_{\substack{\delta \leqslant u \\
\delta \in P_{3}}} r_{1}(\delta) \sum_{s \mid(N, \delta)} \sum_{\substack{k \in P_{1}, k \mid N}} \tau(k) \sum_{n \in A_{1}\left(N /(k s), g_{k s}, a-g(k s), \delta / s\right)} \tau\left(\frac{N /(s k)-n}{\delta} s\right) \\
& +O\left(\frac{N}{\log \mathscr{L}}\right),
\end{aligned}
$$

где $g_{k s}(n)=g(n k s)-g(k s)-$ аддитивная по $n$ функция и

$$
A_{1}(N, g, a, \delta)=\{n: n \in A(N, g, a, \delta),(n, N)=1\} .
$$

Оценим часть последней суммы, в которой $k \geqslant v=\exp \left(10 \log ^{2} \mathscr{L}\right)$. Она будет

$$
\ll \sum_{\delta \leqslant u} r_{1}(\delta) \sum_{s \mid \delta} \sum_{\substack{k \mid N, k \geqslant v}} \tau(k) \frac{N}{\delta k} \mathscr{L}
$$

Пусть $\alpha=\log ^{-1} \mathscr{L}$. Тогда

$$
\sum_{\substack{k \geqslant v, k \mid N}} \frac{\tau(k)}{k} \leqslant \frac{1}{v^{\alpha}} \sum_{k \mid N} \frac{\tau(k)}{k^{1-\alpha}} \ll \exp (-10 \log \mathscr{L}) \exp \left(2 \sum_{p \leqslant \mathscr{L}} \frac{p^{\alpha}}{p}+2 \frac{1}{\mathscr{L}^{1-\alpha}} \frac{\mathscr{L}}{\log \mathscr{L}}\right)
$$

$\ll \exp \left(-10 \log \mathscr{L}+2 \mathrm{e} \log \log \mathscr{L}+2 \mathrm{e}^{\log }{ }^{-1} \mathscr{L}\right) \ll \mathscr{L}^{-9}$.

Следовательно, оцениваемая часть суммы равна $O\left(N \mathscr{L}^{-6}\right)$. Таким образом, имеем

$$
\sup _{a} S(N) \leqslant 4 \sum_{\substack{\delta \leqslant u \\ \delta \in P_{3}}} r_{1}(\delta) \sum_{s \mid(N, \delta)} \sum_{\substack{k \mid N, k \in P_{1}, k \leqslant v}} \tau(k) X\left(\frac{N}{k s}, \frac{\delta}{s}, g_{k s}\right)+O\left(\frac{N}{\log \mathscr{L}}\right)
$$

где $u=\log ^{3} \mathscr{L}, v=\exp \left(10 \log ^{2} \mathscr{L}\right), \mathscr{L}=\log N, g_{k s}(n)=g(k s n)-g(k s)-$ аддитивная по $n$ функция и

$$
X(N, g, \delta):=\sup _{a} \sum_{n \in A_{1}(N, g, a, \delta)} \tau\left(\frac{N-n}{\delta}\right) .
$$


Покажем, что для доказательства теоремы 1 достаточно доказать следующее неравенство:

$$
X(N, g, \delta) \ll \frac{N}{\varphi(\delta) \sqrt{W(N, g)}} \prod_{\substack{p \mid N, p \equiv 1(\bmod 4)}}\left(1-\frac{2}{p}\right)
$$

где

$$
W(N, g)=4+\min _{\lambda}\left(\lambda^{2}+\sum_{p \leqslant N} \frac{1}{p} \min \left(1,(g(p)-\lambda \log p)^{2}\right)\right) .
$$

Учитывая, что $g_{k s}(p)=g(p)$, если $(p, k s)=1$ имеем

$$
W\left(\frac{N}{k s}, g_{k s}\right)=W(N, g)+\Theta\left(\sum_{\frac{N}{u v} \leqslant p \leqslant N} \frac{1}{p}+\sum_{p \mid k s} \frac{1}{p}\right),
$$

где $|\Theta| \leqslant 1$. Следовательно,

$$
\frac{1}{\sqrt{W\left(\frac{N}{k s}, g_{k s}\right)}}-\frac{1}{\sqrt{W(N, g)}} \ll \frac{1}{\sqrt{W(N, g)}} \prod_{p \mid k s}\left(1+\frac{1}{p}\right) .
$$

Поэтому из (4) следует

$$
X\left(\frac{N}{k s}, \frac{\delta}{s}, g_{k s}\right) \ll \frac{N}{k s \varphi\left(\frac{\delta}{s}\right) \sqrt{W(N, g)}} \prod_{\substack{p \mid N, p \equiv 1(\bmod 4)}}\left(1-\frac{2}{p}\right) \cdot \prod_{p \mid k s}\left(1+\frac{3}{p}\right) .
$$

Так как $s \varphi(\delta / s) \geqslant \varphi(\delta)$, то, подставляя последнюю оценку в (3), находим

$$
\begin{aligned}
\sup _{a} S(N) \ll & \frac{N}{\sqrt{W(N, g)}} \prod_{\substack{p \mid N, p \equiv 1(\bmod 4)}}\left(1-\frac{2}{p}\right) \sum_{\delta \in P_{3}} \frac{r_{1}(\delta)}{\varphi(\delta)} \sum_{s \mid \delta} \prod_{p \mid s}\left(1+\frac{3}{p}\right) \\
& \times \sum_{\substack{k \mid N \\
k \in P_{1}}} \frac{\tau(k)}{k} \prod_{p \mid k}\left(1+\frac{3}{p}\right)+\frac{N}{\log \mathscr{L}} \ll \frac{N}{\sqrt{W(N, g)}} .
\end{aligned}
$$

То есть из (4) следует утверждение теоремы 1.

Заметим, что

$$
\frac{1}{\sqrt{W(N, g)}} \prod_{\substack{p \mid N, p \equiv 1(\bmod 4)}}\left(1-\frac{2}{p}\right) \gg(\log \mathscr{L})^{-3 / 2},
$$

и $\delta \leqslant \log ^{3} \mathscr{L}$, поэтому части суммы $X(N, g, \delta)$, равные $O\left(N /\left(\varphi(\delta) \log ^{3 / 2} \mathscr{L}\right)\right)$ или $O\left(N / \log ^{9 / 2} \mathscr{L}\right)$, будут равны $O(R(N, \delta))$, где далее

$$
R(N, \delta)=\frac{N}{\varphi(\delta) \sqrt{W(N, g)}} \prod_{\substack{p \mid N, p \equiv 1(\bmod 4)}}\left(1-\frac{2}{p}\right)
$$

- правая часть соотношения (4). 
Пусть $p(n), g(n)$ - наибольший и наименьший простые делители $n$ соответственно. Представим $n$ в виде $n=n_{1} n_{2}, p\left(n_{1}\right) \leqslant z_{3}, g\left(n_{2}\right)>z_{3}, z_{3}=\exp \left(\mathscr{L}^{\Theta}\right), 1 / 2<\Theta<1$. Рaзобьем $X(n, g, \delta)$ на четыре суммы. В первой сумме $n_{1} \leqslant z_{1}=\exp \left(\mathscr{L}^{\sigma}\right), 0<\sigma<\Theta-1 / 2$, во второй $n_{1}>z_{4}, \log z_{4}=16 \mathscr{L}^{\Theta} \log \mathscr{L}$, в третьей $n_{1} \leqslant z_{4}, n_{2} \leqslant z_{2}=N \mathscr{L}^{-10} /\left(\delta z_{4}\right)$ и в четвертой $z_{1}<n_{1} \leqslant z_{4}, n_{2}>z_{2}$. Пусть $\bar{n}_{1} n_{1} \equiv 1(\bmod d \delta)$. Тогда, применив лемму 4 , получим, что первая сумма

$$
\begin{aligned}
& \ll \sum_{n_{1} \leqslant z_{1}} \sum_{\substack{d \leqslant \sqrt{N / \delta}, d \in P_{1}}} \sum_{\substack{n_{1} n_{2}<N, n_{2} \equiv N \bar{n}_{1}(\bmod d \delta)}} 1 \\
& \ll \sum_{n_{1} \leqslant z_{1}} \sum_{\substack{d \leqslant N, d \in P_{1}}} \frac{N}{n_{1} d \delta \log z_{3}} \ll \frac{N \sqrt{\mathscr{L}} \log z_{1}}{\delta \log z_{3}} \ll R(N, \delta) .
\end{aligned}
$$

Для оценки второй суммы сначала применим неравенство Коши, затем лемму 1. Получим, что она

$$
\leqslant\left(\sum_{n_{2}<N} \sum_{z_{4}<n_{1} \leqslant \frac{N}{n_{2}}} 1\right)^{1 / 2}\left(\sum_{m<N} \tau^{2}(m)\right)^{1 / 2} \ll N \exp \left(-\frac{1}{4} \frac{\log z_{4}}{\log z_{3}}\right) \mathscr{L}^{2} \ll R(N, \delta) .
$$

Аналогично третья сумма будет

$$
\leqslant\left(\sum_{n_{1}<z_{4}} \sum_{n_{2} \leqslant z_{2}} 1\right)^{1 / 2}\left(\sum_{m<N} \tau^{2}(m)\right)^{1 / 2} \ll R(N, \delta) .
$$

Следовательно, имеем

$$
X(N, g, \delta) \leqslant \sup _{a} \sum_{n \in A_{2}(N, g, a, \delta)} \tau\left(\frac{N-n}{\delta}\right)+R(N, \delta)
$$

где

$$
A_{2}(N, g, a, \delta)=\left\{n: n \in A_{1}(N, g, a, \delta), n=n_{1} n_{2}, z_{1}<n_{1} \leqslant z_{4}, n_{2}>z_{2}\right\} .
$$

Избавимся от условия $g(n) \in[a ; a+1)$. С этой целью воспользуемся равенством

$$
\int_{-1}^{1}(1-|t|) \mathrm{e}^{i t u} d t=\left(\frac{\sin (u / 2)}{u / 2}\right)^{2}
$$

и неравенствами

$$
\frac{4}{\pi^{2}} \leqslant\left(\frac{\sin (u / 2)}{u / 2}\right)^{2} \leqslant 1
$$

справедливьми при $|u| \leqslant 2$. Кроме этого от $\tau((N-n) / \delta)$ снова вернемся к $r_{1}((N-n) / \delta)$. Получим

$$
X(N, g, \delta) \leqslant \sup _{a} \frac{\pi^{2}}{4} \sum_{n \in A_{3}(N, \delta)} \int_{-1}^{1}(1-|t|) \mathrm{e}^{i t(g(n)-a)} d t r_{1}\left(\frac{N-n}{\delta}\right)+O(R(N, \delta)),
$$


где

$$
\begin{aligned}
A_{3}(N, \delta)=\left\{n: n=n_{1} n_{2}<N, z_{1}<n_{1} \leqslant z_{4}, n_{2} \geqslant z_{2}\right. \\
\left.(n, N)=1, n \equiv N(\bmod \delta), \frac{N-n}{\delta} \equiv 1(\bmod 4)\right\} .
\end{aligned}
$$

Разобьем $r_{1}(m)$ - сумму $\chi(d)$ по $d \mid m=(N-n) / \delta$ на три суммы. В первой

$$
d<\sqrt{\frac{N}{\delta}} \frac{1}{\Delta}
$$

во второй

$$
\sqrt{\frac{N}{\delta}} \frac{1}{\Delta} \leqslant d<\sqrt{\frac{N}{\delta}} \Delta
$$

и в третьей

$$
d \geqslant \sqrt{\frac{N}{\delta}} \Delta
$$

где $\Delta=\mathscr{L}^{10}$. Если $(N-n) / \delta=d s$ и $d \geqslant \sqrt{N / \delta} \Delta$, то $s \leqslant \sqrt{N / \delta} / \Delta$ и, так как $(N-n) / \delta \equiv 1(\bmod 4)$, то $\chi(d)=\chi(s)$. Следовательно, имеем

$$
r_{1}(m)=2 \sum_{1} \chi(d)+\sum_{2} \chi(d)-\sum_{3} \chi(d)-\sum_{4} \chi(d)
$$

где в $\sum_{1}$ суммирование распространено по $d \mid m, d<\sqrt{N / \delta} / \Delta$, в $\sum_{2}-$ по $d \mid m$, $\sqrt{N / \delta} / \Delta \leqslant d<\sqrt{N / \delta} \Delta$, в $\sum_{3}-$ по

$$
d \mid m, \quad d \in\left[\sqrt{\frac{N}{\delta}} \frac{1}{\Delta} ; \sqrt{\frac{N}{\delta}} \Delta\right) \cap\left[\sqrt{\frac{\delta}{N}} m \Delta ; \sqrt{\frac{N}{\delta}} \Delta\right)
$$

и в $\sum_{4}-$ по

$$
d \mid m, \quad d \in\left[1 ; \sqrt{\frac{N}{\delta}} \frac{1}{\Delta}\right) \cap\left[\sqrt{\frac{\delta}{N}} m \Delta ; \sqrt{\frac{N}{\delta}} \frac{1}{\Delta}\right) .
$$

Возвращаясь к $X(N, g, \delta)$, получим

$$
\begin{aligned}
& X(N, g, \delta) \leqslant \frac{\pi^{2}}{2} \sup _{a} \int_{-1}^{1}(1-|t|) \mathrm{e}^{-i t a} \sum_{d<\sqrt{N / \delta} / \Delta} \chi(d) \sum_{n \in A_{4}(N, \delta, d)} \mathrm{e}^{i t g(n)} d t \\
& +O\left(\sum_{\substack{n<N, n \equiv N(\bmod \delta)}}\left|\sum_{2} \chi(d)\right|+\left|\sum_{5} \chi(d)\right|+\sum_{4} 1\right)
\end{aligned}
$$

где в $\sum_{5}$ суммирование распространено по

$$
\begin{gathered}
d \mid \frac{N-n}{\delta}, \quad \frac{N-n}{\delta} \sqrt{\frac{\delta}{N}} \leqslant d<\sqrt{\frac{N}{\delta}} \Delta, \\
A_{4}(N, \delta, d)=\left\{n: n \in A_{3}(N, \delta), n \equiv N(\bmod d)\right\} .
\end{gathered}
$$


Для оценки первых двух сумм, стоящих под знаком $O(\ldots$ ) применим лемму 6. Получим, что они равны

$$
O\left(\frac{N}{\delta}(\log \mathscr{L})^{5 / 4} \mathscr{L}^{-\beta / 2}\right) \ll R(N, \delta) .
$$

Третья сумма будет

$$
\leqslant \sum_{m<N /\left(\delta \Delta^{2}\right)} \tau(m) \ll \frac{N}{\delta \mathscr{L}^{20}} \cdot \mathscr{L} \ll R(N, \delta) .
$$

Если $n \in A_{4}(N, \delta, d)$, то $n \equiv N(\bmod \delta d)$ и $n \equiv N-\delta(\bmod 4 \delta)$. Пусть $d=d_{1} d_{2}$, $p\left(d_{1}\right) \leqslant z, p\left(d_{2}\right)>z, z=\mathscr{L}^{10}$. Тогда эти два сравнения эквивалентны сравнению $n \equiv N-\delta d_{1} d_{2} \bar{d}_{1} \bar{d}_{2}\left(\bmod 4 \delta d_{1} d_{2}\right)$, где $\bar{d}_{1} d_{1} \equiv 1(\bmod 4), \bar{d}_{2} d_{2} \equiv 1(\bmod 4)$. Заметим, что $(d, 2)=1$. При таком выборе $\bar{d}_{1}, \bar{d}_{2}$ мы имеем $N-\delta d_{1} d_{2} \bar{d}_{1} \bar{d}_{2} \equiv N-\delta d_{1} \bar{d}_{1}\left(\bmod 4 \delta d_{1}\right)$. Так как $(n, N)=1$, то $(\delta d, N)=\left(\delta d, N-\delta d_{1} d_{2} \bar{d}_{1} \bar{d}_{2}\right)=1$. Возможны случаи, когда

1) $N-\delta d_{1} d_{2} \bar{d}_{1} \bar{d}_{2}=M-$ число нечетное,

2) $2 \mid M$, но $4 \nmid M$,

3) $4 \mid M$.

В каждом из этих случаев, поделив обе части сравнения на соответствующую степень двойки, мы можем добиться, чтобы модуль и остаток от деления были взаимно просты. Рассмотрим случай, когда $2 \nmid M$. Напомним, что $\delta \leqslant \log ^{3} \mathscr{L}$, а $z=\mathscr{L}^{10}$, т.е. $\delta<z$. Пусть $Q=\sqrt{N / \delta} / \Delta$. Имеем

$$
\begin{aligned}
& \sum_{\substack{d=d_{1} d_{2} \leqslant Q,(d, N)=1}} \chi(d) \sum_{n \in A_{4}(N, \delta, d)} e^{i t g(n)}=\sum_{\substack{d=d_{1} d_{2} \leqslant Q,(d, N)=1}} \chi\left(d_{1} d_{2}\right) \frac{1}{\varphi\left(d_{2}\right)} \sum_{\substack{n \in A_{4}(N, \delta, d),\left(n, d_{2}\right)=1}} e^{i t g(n)} \\
& +\sum_{\substack{d=d_{1} d_{2} \leqslant Q,(d, N)=1}} \chi(d)\left(\sum_{\substack{z_{1}<n_{1} \leqslant z_{4}, z_{2}<n_{2}<N / n_{1}, n_{1} n_{2} \equiv N-\delta d_{1} d_{2} \bar{d}_{1} \bar{d}_{2}\left(\bmod 4 \delta d_{1} d_{2}\right)}} e^{i t g\left(n_{1} n_{2}\right)}\right. \\
& \left.-\frac{1}{\varphi\left(d_{2}\right)} \sum_{\substack{z_{1}<n_{1} \leqslant z_{4}, z_{2}<n_{2}<N / n_{1}, n_{1} n_{2} \equiv N-\delta d_{1} \bar{d}_{1}\left(\bmod 4 \delta d_{1}\right) \\
\left(n_{1} n_{2}, d_{2}\right)=1}} e^{i t g\left(n_{1} n_{2}\right)}\right) .
\end{aligned}
$$

Используя лемму 7 , выбрав в ней $A=20$, получим, что вторая сумма в правой части последнего равенства

$$
\begin{aligned}
\ll & \frac{\sqrt{N}}{\mathscr{L}^{10}}\left(\sqrt{N \delta} \frac{1}{\mathscr{L}^{10}}+\sqrt{N} \mathscr{L}^{3 / 2}\right)+\log \mathscr{L}\left(\sqrt{N \delta} \frac{1}{\mathscr{L}} \sqrt{N} \mathscr{L}+\left(\frac{N}{\sqrt{z_{2}}}+\frac{N}{\sqrt{z_{1}}}\right) \mathscr{L}^{2}\right. \\
& \left.+\frac{N}{z} \mathscr{L} \log \frac{N}{z_{1} z_{2}}\right) \ll \frac{N}{\delta \mathscr{L}} \ll R(N, \delta) .
\end{aligned}
$$

Подставляя полученные оценки в (5), находим

$$
\begin{aligned}
X(N, g, \delta) \leqslant \sup _{a} \frac{\pi^{2}}{2} \int_{-1}^{1}(1-|t|) \mathrm{e}^{-i t a} \sum_{\substack{d_{1} \leqslant \sqrt{N / \delta} / \Delta\\
}} \chi\left(d_{1}\right) \\
\times \sum_{n \in A_{4}\left(N, \delta, d_{1}\right)} \mathrm{e}^{i t g(n)} \sum_{\substack{d_{2} \leqslant \sqrt{N / \delta} /\left(\Delta d_{1}\right),\left(d_{2}, N n\right)=1}} \frac{\chi\left(d_{2}\right)}{\varphi\left(d_{2}\right)} d t+O(R(N, \delta)) .
\end{aligned}
$$


Если $q(m)>z, \log m=O(z)$, то

$$
\frac{m}{\varphi(m)}=1+O\left(\exp \left(\sum_{\substack{p \mid m, p>z}} \frac{1}{p}\right)-1\right)=1+O\left(\frac{\log m}{z}\right) .
$$

Можно считать, что в правой части $(6) d_{1} \leqslant z_{5}=\exp \left(50 \log ^{2} \mathscr{L}\right)$. Действительно, часть суммы по $d_{1}>z_{5}$ в силу леммы 2 будет

$$
\ll \frac{N}{\delta} \mathscr{L} \sum_{z_{5}<d_{1} \leqslant N} \frac{1}{d_{1}} \ll \frac{N}{\delta} \mathscr{L} \log \mathscr{L} \exp \left(-\frac{1}{2} \frac{\log z_{5}}{\log z}\right) \ll R(N, \delta) .
$$

Следовательно, используя лемму 3 , имеем

$$
\sum_{\substack{d_{2} \leqslant \sqrt{N / \delta} / \Delta,\left(d_{2}, N n\right)=1}} \frac{\chi\left(d_{2}\right)}{\varphi\left(d_{2}\right)}=\sum_{\substack{d_{2} \leqslant \sqrt{N / \delta} / \Delta,\left(d_{2}, N n\right)=1}} \frac{\chi\left(d_{2}\right)}{d_{2}}+O\left(\frac{1}{\mathscr{L}^{8}}\right)=1+O\left(\frac{1}{\delta \mathscr{L}}\right)
$$

и поэтому

$$
X(N, g, \delta) \leqslant \sup _{a} \frac{\pi^{2}}{2} \int_{-1}^{1}(1-|t|) e^{-i t a} \sum_{\substack{d \leqslant z_{5}, p(d) \leqslant z}} \chi(d) \sum_{n \in A_{5}(N, \delta, d)} e^{i t g(n)} d t+R(N, \delta),
$$

где

$$
A_{5}(N, \delta d)=\{n: n<N,(n, N)=1, n \equiv N(\bmod \delta d)\} .
$$

Здесь мы заменим условие $n \in A_{4}(N, \delta, d)$ на $n \in A_{5}(N, \delta d) \supset A_{4}(N, \delta, d)$. Это можно сделать, так как

$$
\sum_{\substack{d \mid m, p(d) \leqslant z}} \chi(d) \geqslant 0 \text { и } \int_{-1}^{1}(1-|t|) e^{i t(g(n)-a)} d t \geqslant 0 .
$$

Условие $d \leqslant z_{5}$, как мы видели ранее (см. (7)), можно отбросить.

Чтобы уменьшить промежуток изменения $d$, мы еще раз повторим предыдущие рассуждения. Представим $n$ в виде $n=n_{1} n_{2}$, где $p\left(n_{1}\right) \leqslant z_{3}^{\prime}=\exp \left(\log ^{5} \mathscr{L}\right), q\left(n_{2}\right)>z_{3}^{\prime}$. Пусть $z_{1}^{\prime}=\exp \left(\log ^{2} \mathscr{L}\right), z_{4}^{\prime}=\exp \left(\log ^{15} \mathscr{L}\right)$ и разобьем сумму по $n$ на четыре суммы. В первой сумме $n_{1} \leqslant z_{1}$, во второй $n_{1}>z_{4}^{\prime}$, в третьей $n_{1} \leqslant z_{4}^{\prime}, n_{2} \leqslant z_{2}^{\prime}=N /\left(\delta z_{4}^{\prime} \mathscr{L}^{10}\right)$, в четвертой $z_{1}^{\prime}<n_{1} \leqslant z_{4}^{\prime}, n_{2}>z_{2}^{\prime}$. Тогда сумма, стояшая под интегралом в правой части (8) также разобьется на четыре суммы. Применив лемму 4 , получим, что первая сумма будет

$$
\ll \sum_{p(d) \leqslant z} \sum_{n_{1} \leqslant z_{1}^{\prime}} \frac{N}{\varphi(\delta d) n_{1} \log z_{3}^{\prime}} \ll \frac{N}{\varphi(\delta)} \log ^{-2} \mathscr{L} \ll R(N, \delta) .
$$

Используя лемму 1 , получим, что вторая и третья суммы

$$
\ll z_{5} N \exp \left(-\frac{1}{2} \frac{\log z_{4}^{\prime}}{\log z_{3}^{\prime}}\right) \cdot \mathscr{L}+\frac{N}{\delta \mathscr{L}^{10}} \cdot \log z_{5} \ll R(N, \delta) .
$$


Пусть $z^{\prime}=\log ^{20} \mathscr{L}$. Представим $d$ в виде $d=d_{1} d_{2}, p\left(d_{1}\right) \leqslant z^{\prime}, z^{\prime}<q\left(d_{2}\right), p\left(d_{2}\right) \leqslant z$. Тогда четвертую сумму можно представить в следующем виде

$$
\begin{aligned}
& \sum_{\substack{d=d_{1} d_{2} \leqslant z_{5},\left(d_{2}, N\right)=1}} \chi\left(d_{1}\right) \frac{\chi\left(d_{2}\right)}{\varphi\left(d_{2}\right)} \sum_{\substack{n \in A_{6}\left(N, \delta d_{1}\right),\left(n, d_{2}\right)=1}} e^{i t g(n)} \\
& \quad+\sum_{\substack{d=d_{1} d_{2} \leqslant z_{5} \\
\left(d_{2}, N\right)=1}}\left(\sum_{n \in A_{6}(N, \delta d)} e^{i t g(n)}-\frac{1}{\varphi\left(d_{2}\right)} \sum_{\substack{n \in A_{6}\left(N, \delta d_{1}\right),\left(n, d_{2}\right)=1}} e^{i t g(n)}\right),
\end{aligned}
$$

где

$$
A_{6}(N, m)=\left\{n: n \in A_{5}(N, m), n=n_{1} n_{2}, z_{1}^{\prime}<n_{1} \leqslant z_{4}^{\prime}, n_{2}>z_{2}^{\prime}\right\} .
$$

Используя лемму 7 с $Q=z_{5} u, A=20, z_{1}=z_{1}^{\prime}, z_{2}=z_{2}^{\prime}, z=z^{\prime}$, получим, что вторая сумма

$$
\ll \frac{N}{\mathscr{L}}+\frac{N \log z_{5} u}{\log ^{22} \mathscr{L}} \log \frac{N}{z_{1}^{\prime} z_{2}^{\prime}} \ll N \frac{\log ^{17} \mathscr{L}}{\log ^{22} \mathscr{L}} \ll R(N, \delta) .
$$

В первой сумме перейдем снова к $n \in A_{5}\left(N, \delta d_{1}\right)$. Кроме этого, можно считать, что $d_{1} \leqslant z_{5}^{\prime}=\exp \left((\log \log \mathscr{L})^{3}\right)$. Действительно, часть суммы по $d_{1}>z_{5}^{\prime}$ в силу леммы 2 будет

$$
\ll \sum_{\substack{d_{1}>z_{5}^{\prime}, p\left(d_{1}\right) \leqslant z^{\prime}}} \frac{N}{\delta d_{1}} \frac{\log z}{\log z^{\prime}} \ll \frac{N}{\delta} \exp \left(-\frac{1}{2} \frac{\log z_{5}^{\prime}}{\log z^{\prime}}\right) \log z \ll R(N, \delta) .
$$

Далее, используя второе неравенство леммы 2, имеем

$$
\begin{aligned}
& \sum_{\substack{d_{2} \leqslant z_{5} / d_{1},\left(d_{2}, N n\right)=1}} \frac{\chi\left(d_{2}\right)}{\varphi\left(d_{2}\right)}=\prod_{z<p \leqslant z^{\prime}}\left(1+\frac{\chi(p) p}{(p-1)(p-\chi(p))}\right) \cdot \prod_{p \mid N}\left(1+\frac{\chi(p) p}{(p-1)(p-\chi(p))}\right)^{-1} \\
& \times\left(1+O\left(\sum_{\substack{d_{2} \mid n \\
d_{2}>z^{\prime}}} \frac{1}{d_{2}}\right)\right)+\exp \left(-\frac{1}{5} \frac{\log z_{5}}{\log z^{\prime}}\right) \log ^{2} z^{\prime}
\end{aligned}
$$

Заметим, что

$$
\begin{aligned}
& \prod_{z^{\prime}<p \leqslant z}\left(1+\frac{\chi(p) p}{(p-1)(p-\chi(p))}\right) \ll 1, \\
& \prod_{\substack{p \mid N, z^{\prime}<p \leqslant z}}\left(1+\frac{\chi(p) p}{(p-1)(p-\chi(p))}\right)^{-1} \ll \prod_{\substack{p \mid N, p>z^{\prime}}}\left(1-\frac{\chi(p)}{p}\right) .
\end{aligned}
$$

Кроме этого, имеем

$$
\sum_{d_{1} \leqslant z_{5}} \sum_{n \in A_{6}\left(N, \delta d_{1}\right)} \sum_{\substack{d_{2} \mid n, z^{\prime}<d_{2} \leqslant z_{5}}} \frac{1}{d_{2}} \ll \sum_{d_{1} \leqslant z_{5}} \frac{N}{\delta d_{1}} \sum_{d_{2}>z^{\prime}} \frac{1}{d_{2}^{2}} \ll R(N, \delta) .
$$


Поэтому возвращаясь к соотношению (8), получим

$$
X(N, g, \delta) \leqslant \prod_{\substack{p \mid N \\ p>z^{\prime}}}\left(1-\frac{\chi(p)}{p}\right)\left|X_{1}(N, g, \delta)\right|+R(N, \delta)
$$

где

$$
X_{1}(N, g, \delta)=\int_{-1}^{1} \sum_{\substack{d \leqslant z_{5}^{\prime}, p(d) \leqslant z^{\prime}}} \chi(d) \sum_{\chi_{\delta d}} \bar{\chi}_{\delta d}(N) \frac{1}{\varphi(\delta d)} \sum_{\substack{n<N,(n, N)=1}} \chi_{\delta d}(n) e^{i t g(n)}(1-|t|) e^{-i t a} d t .
$$

Напомним, что $z^{\prime}=\log ^{22} \mathscr{L}, z_{5}^{\prime}=\exp \left((\log \log \mathscr{L})^{3}\right), \delta \leqslant \log ^{3} \mathscr{L}, \mathscr{L}=\log N$, $\delta d \leqslant \log ^{3} \mathscr{L} \exp \left((\log \log \mathscr{L})^{3}\right), p(\delta d) \leqslant \log ^{22} \mathscr{L}=z^{\prime}$.

Разобьем $[-1 ; 1]$ на два множества $U_{1}$ и $U_{2}$. Для $t \in U_{1}$ найдутся $\lambda(t)=\lambda,|\lambda| \leqslant T=$ $\exp \left((\log \log \mathscr{L})^{4}\right)$ и характер Дирихле $\chi_{m}, m=m(t) \leqslant T, p(m) \leqslant z^{\prime}$, такие, что

$$
\sum_{p<N} \frac{1}{p}\left(1-\operatorname{Re} \chi_{m}(p) e^{i t g(p)} p^{-i \lambda}\right) \ll(\log \log \mathscr{L})^{4}
$$

Множество $U_{2}=[-1 ; 1] \backslash U_{1}$. Применив лемму 8, получим

$$
\int_{U_{2}} \sum_{d \leqslant z_{5}^{\prime}} \frac{1}{\varphi(\delta d)} \sum_{\chi_{\delta d}}\left|\sum_{\substack{n<N,(n, N)=1}} \chi_{\delta d}(n) e^{i t g(n)}\right| d t \ll N z_{5}^{\prime} \exp \left(-\frac{1}{2}(\log \log \mathscr{L})^{4}\right) \ll R(N, \delta) .
$$

Таким образом, задача свелась к оценке интеграла по $U_{1}$. Обозначим через $l=l(t)$ модуль примитивного характера порождающего $\chi_{m}$, с которым выполняется неравенство (11). Очевидно, что (11) справедливо и для $\chi_{l}^{*}$. Применив леммы 9,8, получим, что для всех характеров $\chi_{\delta d}$, не порожденных $\chi_{l}^{*}$, справедливо неравенство

$$
\left|\sum_{\substack{n<N,(n, N)=1}} \chi_{\delta d} e^{i t g(n)}\right| \ll N \mathscr{L}^{-1 / 4}
$$

Поэтому в сумме правой части (10) по характерам $\chi_{\delta d}$ можно оставить только характеры, порожденные $\chi_{l}^{*}$. Пусть $l=l_{1} l_{2}$, где $\delta=l_{1} \delta_{1},\left(\delta_{1}, l_{2}\right)=1$. Тогда если $\left(n, \delta d_{1}\right)=1$, то $\chi_{\delta l_{2} d_{1}}(n)=\chi \delta l_{2}(n)$. Таким образом, имеем

$$
\begin{aligned}
X_{1}(N, g, \delta)= & \int_{U_{1}} \bar{\chi}_{\delta l_{2}}(N) \sum_{\substack{n<N,(n, N)=1}} \chi_{\delta l_{2}}(n) \sum_{\begin{array}{c}
d_{1} \leqslant z_{5} / l_{2}, \\
p\left(l_{2} d_{1}\right)<z^{\prime} \\
\left(d_{1}, n N\right)=1
\end{array}} \frac{\chi\left(\delta l_{2} d_{1}\right)}{\varphi\left(\delta l_{2} d_{1}\right)} e^{i t g(n)}(1-|t|) e^{-i t a} d t \\
& +O(R(N, \delta)) .
\end{aligned}
$$

Для тех $t \in U_{1}$, для которых $l_{2} \geqslant \log ^{3} \mathscr{L}$, модуль подынтегрального выражения будет

$$
\ll \frac{N}{\varphi(\delta)}(\log \mathscr{L})^{-1}(\log \log \mathscr{L})^{3} \ll R(N, \delta),
$$


поэтому можно считать, что $l_{2} \leqslant \log ^{3} \mathscr{L}$. Используя лемму 2 и учитьвая, что $(n, N)=1$ и $\left(\delta l_{2}, n N\right)=1$, имеем

$$
\begin{aligned}
\sum_{\substack{p \leqslant z_{5}^{\prime} \\
d_{1} \leqslant l_{2},\left(d_{1}\right)<z^{\prime},\left(d_{1}, n N\right)=1}} \frac{\chi\left(d_{1}\right)}{\varphi\left(\delta l_{2} d_{1}\right)}= & \frac{1}{\varphi\left(\delta l_{2}\right)} \prod_{p \leqslant z^{\prime}}\left(1+\frac{\chi(p) p}{(p-1)(p-\chi(p))}\right) \prod_{p \mid \delta l_{2}} \frac{p(p-1)}{p^{2}-p+\chi(p)} \\
& \times \prod_{\substack{p \mid n N \\
p \leqslant z^{\prime}}}\left(1-\frac{\chi(p) p}{p^{2}-p+\chi(p)}\right)+O\left(\frac{1}{\varphi\left(\delta l_{2}\right) \log ^{2} \mathscr{L}}\right) .
\end{aligned}
$$

Возвращаясь к соотношениям (9) и (10), находим

$$
X(N, g, \delta) \ll \prod_{p \mid N}\left(1-\frac{\chi(p)}{p}\right)\left|X_{2}(N, g, \delta)\right|+R(N, \delta),
$$

где

$$
\begin{gathered}
X_{2}(N, g, \delta)=\int_{U_{1}} \frac{1}{\varphi\left(\delta l_{2}\right)} \bar{\chi}_{\delta l_{2}}(N) \prod_{p \mid \delta l_{2}} \frac{p(p-1)}{p^{2}-p+\chi(p)}, \\
\sum_{\substack{n<N,(n, N)=1}} \chi_{\delta l_{2}}(n) e^{i t g(n)} F(n)(1-|t|) e^{-i t a} d t \\
F(n)=\prod_{\substack{p \mid n, p \leqslant z^{\prime}}}\left(1+\frac{s(p)}{\sqrt[3]{p}}\right), \quad s(p)=-\frac{p \sqrt[3]{p} \chi(p)}{p^{2}-p+\chi(p)}
\end{gathered}
$$

Имеем

$$
\begin{aligned}
& \sum_{\substack{n<N,(n, N)=1}} \chi_{\delta l_{2}}(n) F(n) e^{i t g(n)} \\
& \quad=\sum_{\substack{d \leqslant \log ^{9} \mathscr{L}, p(d) \leqslant z^{\prime}}} \chi_{\delta l_{2}}(d) e^{i t g(d)} \frac{s(d)}{\sqrt[3]{d}} \sum_{\substack{n<N,(n, N)=1}} \chi_{\delta l_{2}}(n) e^{i t g_{d}(n)}+O\left(\log ^{-2} \mathscr{L}\right),
\end{aligned}
$$

где $s(d)=\prod_{p \mid d} s(p), g_{d}(n)=g(n d)-g(d)$ - аддитивная по $n$ функция. Из неравенства (11) следует, что

$$
\sum_{p<N / d} \frac{1}{p}\left(1-\operatorname{Re} \chi_{\delta l_{2}}(p) e^{i t g_{d}(p)} p^{-i \lambda}\right) \leqslant(\log \log \mathscr{L})^{4}+O(\log \log \mathscr{L}) .
$$

Применив суммирование по Абелю, получим

$$
\begin{aligned}
\sum_{\substack{n<N / d,(n, N)=1}} e^{i t g_{d}(n)} \chi_{\delta l_{2}}(n)= & \sum_{\substack{n<N / d,(n, N)=1}} e^{i t g_{d}(n)} \chi_{\delta l_{2}}(n) n^{-i \lambda} \mathrm{e}^{i \lambda \log N} \\
& -i \lambda \int_{1}^{N / d} \sum_{\substack{n<u,(n, N)=1}} e^{i t g_{d}(n)} \chi_{\delta l_{2}}(n) n^{-i \lambda} e^{i \lambda \log u} \frac{d u}{u}
\end{aligned}
$$


Учитывая, что $|\lambda| \leqslant \exp \left((\log \log \mathscr{L})^{4}\right)$ и, поэтому, из (14) следует

$$
\sum_{p<N / d} \frac{1}{p}\left|1-\chi_{\delta l_{2}}(p) e^{i t g_{d}(p)} p^{-i \lambda}\right| \leqslant 2 \sqrt{\log \mathscr{L}}(\log \log \mathscr{L})^{2}
$$

а также используя лемму 10, получим

$$
\left|\sum_{\substack{n<N / d,(n, N)=1}} e^{i t g_{d}(n)} \chi_{\delta l_{2}}(n)-\frac{\varphi(N)}{N} \sum_{n<N / d} e^{i t g_{d}(n)} \chi_{\delta l_{2}}(n)\right| \ll \frac{N}{d} \mathscr{L}^{-1 / 5} .
$$

Следовательно, соотношения (12) и (13) примут вид

$$
X(N, g, \delta) \ll \prod_{p \mid N}\left(1-\frac{\chi(p)+1}{p}\right)\left|X_{3}(N, g, \delta)\right|+R(N, \delta),
$$

где

$$
X_{3}(N, g, \delta)=\int_{U_{1}} \frac{1}{\varphi\left(\delta l_{2}\right)} \bar{\chi}_{\delta l_{2}}(N) \Pi\left(\delta l_{2}\right) \sum_{n<N} \chi_{\delta l_{2}}(n) e^{i t g(n)} F(n)(1-|t|) e^{-i t a} d t
$$

Здесь

$$
\Pi(m)=\prod_{p \mid m} \frac{p(p-1)}{p^{2}-p+\chi(p)} .
$$

Для доказательства теоремы 1 теперь достаточно показать, что

$$
X_{3}(N, g, \delta) \ll \frac{N}{\varphi(\delta) \log \mathscr{L}} .
$$

Вспоминая определение $F(n)$, имеем

$$
\left|X_{3}(N, g, \delta)\right| \ll \frac{1}{\varphi(\delta)} \sum_{d \leqslant \log ^{3} \mathscr{L}} \frac{1}{\sqrt[3]{d}}\left|X_{4}\left(\frac{N}{d}, g_{d}, \delta\right)\right|+\frac{N}{\varphi(\delta) \log \mathscr{L}}
$$

где

$$
\left|X_{4}\left(\frac{N}{d}, g_{d}, \delta\right)\right| \leqslant \int_{U_{1}}\left|\sum_{n<N / d} e^{i t g_{d}(n)} \chi_{\delta l_{2}}(n)\right| d t
$$

Дальнейшие рассуждения почти полностью повторяют заключительную часть доказательства теоремы 1 [4]. Дополнительную сложность вносит наличие суммы по $d \leqslant \log ^{3} \mathscr{L}$. Рассмотрим случай $d=1$. Применив лемму 8, получим

$$
X_{4}(N, g, \delta) \ll N \int_{-1}^{1} \exp \left(-\frac{1}{2} \min _{\chi_{\Delta}, \lambda} \sum_{p<N} \frac{1}{p}\left(1-\operatorname{Re} e^{i \operatorname{tg}(p)} p^{-i \lambda} \chi_{\delta}(p)\right)\right) d t+\frac{N}{\log \mathscr{L}}
$$


где минимум берется по $|\lambda| \leqslant \log ^{2} \mathscr{L}$ и $\chi_{\Delta}, \Delta \leqslant \log ^{6} \mathscr{L}$. Напомним, что $\delta \leqslant \log ^{3} \mathscr{L}$ и $l_{2} \leqslant \log ^{3} \mathscr{L}$. Обозначим

$$
\begin{gathered}
M(t)=\min _{\chi_{\Delta}, \lambda} \sum_{p<N} \frac{1}{p}\left(1-\operatorname{Re} e^{i t g(p)} p^{-i \lambda} \chi_{\Delta}(p)\right), \\
E_{k}=\{t: t \in[-1 ; 1], M(t) \leqslant k\}, \quad k=1,2, \ldots, \quad K=4[\log \log \mathscr{L}] .
\end{gathered}
$$

Тогда имеем

$$
X_{4}(N, g, \delta) \ll N \sum_{1 \leqslant k \leqslant K} \exp \left(-\frac{k}{2}\right) m\left(E_{k}\right)+N(\log \mathscr{L})^{-1} .
$$

Предположим, что для всех $1 \leqslant k \leqslant K$ вьполняется неравенство

$$
m\left(E_{k}\right) \leqslant \frac{200 k}{\sqrt{\log \mathscr{L}}} .
$$

Тогда мы получим

$$
X_{4}(N, g, \delta) \ll N \sum_{k \geqslant 1} \exp \left(-\frac{k}{2}\right) k \log ^{-1 / 2} \mathscr{L} \ll N \log ^{-1 / 2} \mathscr{L}
$$

Аддитивные функции $g_{d}(n)$ и $g(n)$ отличаются только на $p \mid d$, поэтому применив лемму 8, получим, что в этом случае для всех $d \leqslant \log ^{3} \mathscr{L}$ имеем

$$
\begin{aligned}
X_{4} & \left(\frac{N}{\delta}, g_{d}, \delta\right) \\
& \ll \frac{N}{d} \int_{-1}^{1} \exp \left(-\frac{1}{2} \min _{\chi_{\Delta}, \lambda} \sum_{p<N / d} \frac{1}{p}\left(1-\operatorname{Re} e^{i t g_{d}(p)} p^{-i \lambda} \chi_{\Delta}(p)\right)\right) d t+\frac{N}{d \log \mathscr{L}} \\
& \ll \frac{N}{d} \prod_{p \mid d}\left(1+\frac{1}{p}\right) \int_{-1}^{1} \exp \left(-\frac{1}{2} M(t)\right) d t+\frac{N}{d \log \mathscr{L}} \ll \frac{N \tau(d)}{d} \log ^{-1 / 2} \mathscr{L} .
\end{aligned}
$$

Следовательно,

$$
X_{3}(N, g, \delta) \ll \frac{1}{\varphi(\delta)} \sum_{d \leqslant \log ^{3} \mathscr{L}} \frac{N \tau(d)}{d^{2}} \log ^{-1 / 2} \mathscr{L} \ll \frac{N}{\varphi(\delta) \sqrt{W(N)}} .
$$

Предположим, что найдется $1 \leqslant k \leqslant K$, для которого

$$
m\left(E_{k}\right)>\frac{200 k}{\sqrt{\log \mathscr{L}}} .
$$

Множество $E_{k}$ симметрично относительно нуля и содержит нуль, поэтому (см., например, лемму $5.3[1])$ каждое число $t \in[-1 ; 1]$ представимо в виде $t_{1}+\cdots+t_{r}$, где $t_{j} \in E_{k}$ и $r=\left[12 / m\left(E_{k}\right)\right]$. Напомним, что если $t_{j} \in E_{k}$, то найдется $\lambda_{i},\left|\lambda_{j}\right| \leqslant \log ^{2} \mathscr{L}$, и $\chi \Delta_{j}, \Delta_{j} \leqslant \log ^{6} \mathscr{L}$, такие, что справедливо неравенство

$$
\sum_{p<N} \frac{1}{p}\left(1-\operatorname{Re} e^{i t_{j} g(p)} \chi_{\Delta_{j}}(p) p^{-i \lambda_{j}}\right) \leqslant k, \quad j=1, \ldots, r .
$$


Пусть $z_{j}, j=1, \ldots, r,-$ комплексные числа, $\left|z_{j}\right| \leqslant 1$. Тогда (см., например, [4]) по индукции можно доказать следующее неравенство:

$$
1-\operatorname{Re} z_{1} \ldots z_{r} \leqslant r^{2} \sum_{j=1}^{r}\left(1-\operatorname{Re} z_{j}\right)
$$

Положим $t=t_{1}+\cdots+t_{r}, \lambda=\lambda_{1}+\cdots+\lambda_{r}, \chi_{\Delta}=\chi_{d_{1}} \cdots \chi_{d_{r}}$. Из предыдущих двух неравенств следует, что для любого $t \in[-1 ; 1]$ сушествует $\lambda=\lambda(t)$ и $\chi \Delta$ такие, что

$$
\sum_{p<N} \frac{1}{p}\left(1-\operatorname{Re} e^{i t g(p)} \chi_{\Delta}(p) p^{-i \lambda}\right) \leqslant r^{2} k \leqslant 13^{2} \frac{\log \mathscr{L}}{200^{2} k} \ll \frac{\log \mathscr{L}}{102} .
$$

Заметим, что $|\lambda| \leqslant r \log ^{2} \mathscr{L} \leqslant \log ^{3} \mathscr{L}, \Delta(t) \leqslant \log ^{6 r} \mathscr{L} \leqslant \log ^{6} \sqrt{\log \mathscr{L}} \mathscr{L}$ и $p(\Delta) \leqslant$ $\log ^{6} \mathscr{L}$. Покажем, что $\chi_{\Delta(t)}$ - главньй характер. Рассмотрим любые $t_{1}, t_{2} \in[-1 ; 1]$ такие, что $t_{1}+t_{2} \in[-1 ; 1]$. Докажем, что характер $\chi_{m}=\chi_{\Delta\left(t_{1}+t_{2}\right)} \bar{\chi}_{\Delta\left(t_{1}\right)} \bar{\chi}_{\Delta\left(t_{1}\right)}{ }^{-}$ главный характер. Используя неравенства (18) и (17), имеем

$$
\sum_{p<N} \frac{1}{p}\left(1-\operatorname{Re} \chi_{m}(p) p^{-i\left(\lambda\left(t_{1}+t_{2}\right)-\lambda\left(t_{1}\right)-\lambda\left(t_{2}\right)\right)}\right) \leqslant 9 \frac{\log \mathscr{L}}{102} \ll \frac{\log \mathscr{L}}{10}
$$

Положим $\sigma_{N}=1+1 / \log N$. Тогда левая часть неравенства равна

$$
\log \zeta\left(\sigma_{N}\right)-\log \left|L\left(\sigma_{N}+i\left(\lambda\left(t_{1}+t_{2}\right)-\lambda\left(t_{1}\right)-\lambda\left(t_{2}\right)\right), \chi_{m}\right)\right|+O(1)
$$

Если $\chi_{m}-$ не главньй характер, то она будет равна (см., например, [7])

$\log \mathscr{L}+O(1)+O\left(\log |\Delta|\left(\left|\lambda\left(t_{1}+t_{2}\right)-\lambda\left(t_{1}\right)-\lambda\left(t_{2}\right)\right|+2\right)\right)=\log \mathscr{L}+O(\sqrt{\log \mathscr{L}} \log \log \mathscr{L})$

То есть получим противоречие. Таким образом, $\chi_{d\left(t_{1}+t_{2}\right)} \bar{\chi}_{d\left(t_{1}\right)} \bar{\chi}_{d\left(t_{2}\right)}-$ главньй характер. Положим

$$
H=\left(\prod_{p \leqslant \log ^{6} \mathscr{L}} p\right)^{s}, \quad \text { где } s \leqslant \sqrt{\log \mathscr{L}} .
$$

Тогда модули всех характеров, о которых шла речь вьше, будут делить $H$. Обозначим через $\tilde{\chi}_{\Delta}$ характер по модулю $H$, порожденньй $\chi_{\Delta}$. Тогда для любых $t_{1}, t_{2} \in[-1 ; 1]$, $t_{1}+t_{2} \in[-1 ; 1]$, имеем $\widetilde{\chi}_{\Delta\left(t_{1}+t_{2}\right)}=\tilde{\chi}_{\Delta\left(t_{1}\right)} \cdot \widetilde{\chi}_{\Delta\left(t_{2}\right)}$ и, поэтому, $\widetilde{\chi}_{\Delta(t)}=\left(\widetilde{\chi}_{\Delta(t / h)}\right)^{h}=\tilde{\chi}_{0}$, где $h=\varphi(H)$. Мы доказали, что $\tilde{\chi}_{\Delta(t)}$-главньй характер. Учитьвая, что $\Delta(t) \mid H$ и

$$
\sum_{p \mid H} \frac{1}{p} \leqslant 12 \log \log \log \mathscr{L}
$$

используя неравенство (18), получим, что для любого $t \in[-1 ; 1]$ существует $\lambda=\lambda(t)$, $|\lambda| \leqslant \log ^{3} \mathscr{L}$, такое, что

$$
\sum_{p<N} \frac{1}{p}\left(1-\operatorname{Re} e^{i t g(p)} p^{-i \lambda}\right) \leqslant \frac{\log \mathscr{L}}{100}
$$


Из леммы 9 следует, что примитивный характер $\chi_{l(t)}^{*}$, порождающий характеры $\chi_{\delta d}$, будет главньй, т.е. $l=l_{1} \cdot l_{2}=1$ и соотношение $(15)$ примет вид

$$
X_{3}(N, g, \delta)=\frac{1}{\varphi(\delta)} \bar{\chi}_{\delta}(N) \Pi(\delta) \int_{-1}^{1} \sum_{\substack{n<N,(n, \delta)=1}} e^{i t g(n)} F(n)(1-|t|) e^{-i t a} d t+O(R(N, \delta))
$$

Следовательно, получим

$$
\begin{aligned}
X_{3}(N, g, \delta) & \ll \frac{1}{\varphi(\delta)} \sum_{d \leqslant \log ^{9} \mathscr{L}} \frac{s(d)}{\sqrt[3]{d}} \sum_{n<N / d}\left(\frac{\sin \left(g_{d}(n)-a\right) / 2}{\left(g_{d}(n)-a\right) / 2}\right)^{2}+\frac{N}{\varphi(d) \log ^{3} \mathscr{L}} \\
& \ll \frac{1}{\varphi(\delta)} \sum_{d \leqslant \log ^{9} \mathscr{L}} \frac{s(d)}{\sqrt[3]{d}} \sup _{a}\left|\left\{n: n<\frac{N}{d}, g_{d}(n) \in[a ; a+1)\right\}\right|+\frac{N}{\varphi(d) \log ^{3} \mathscr{L}} .
\end{aligned}
$$

Учитывая, что $g_{d}(p) \neq g(n)$ только для $p \mid d$, и используя лемму 11 , получим

$$
X_{3}(N, g, \delta) \ll \frac{1}{\varphi(\delta)} \sum_{d \leqslant \log ^{9} \mathscr{L}} \frac{s(d)}{\sqrt[3]{d} d} \cdot \frac{N}{\sqrt{W(N)}} \sum_{p \mid d} \frac{1}{p} \ll \frac{N}{\varphi(\delta) \sqrt{W(N)}} .
$$

Из этой оценки, как отмечалось ранее, вытекает утверждение теоремы 1.

\section{СПИСОК ЦИТИРОВАННОЙ ЛИТЕРАТУРЫ}

[1] Ruzsa I. On the concentration of additive functions // Acta Math. Aca. Sci. Hungar. 1980. V. 36. P. $215-232$.

[2] Elliott P.D.T.A. The concentration function of additive functions on shifted primes // Acta Math. 1994. V. 173. P. 1-35.

[3] Тимофеев Н. М. Гипотеза Эрдеша-Кубилюса о распределении значений аддитивных функций на последовательности сдвинутых простых чисел // Acta Arithmetica. 1991. Т. 58. № 2. C. 113-131.

[4] Тимофеев Н. М., Хрипунова М. Б. О функции концентрации аддитивной функции с немультипликативньм весом // Матем. заметки. 2004. Т. 75. №6. С. 877-894.

[5] Тимофеев Н. М. Проблема Харди и Литтлвуда для чисел, имеющих заданное число простых делителей // Изв. РАН. Сер. матем. 1995. Т. 59. №6. С. 181-206.

[6] Tenenbaum G. Introduction à la théorie analytique et probabiliste des nombres. Institut Elie Cartan, 13. Université de Nancy I, 1990.

[7] Карацуба А. А. Основы аналитической теории чисел. 2-е изд. М.: Наука, 1983.

[8] Halberstam H., Richert H. E. Sieve Methods. London-New York: Academic Press, 1974.

[9] Hall R., Tenenbaum G. 90 Divisors. Cambridge: Cambridge University Press, 1988.

[10] Хооли К. Применение методов решета в теории чисел. М.: Наука, 1987. 